\title{
Successful management of arteriovenous fistula after coronary catheterization via the snuffbox approach
}

\author{
Lukasz Kozinski ${ }^{1}$, Alicja Dabrowska-Kugacka ${ }^{2}$, Zbigniew Orzalkiewicz $^{1}$ \\ ${ }^{1}$ Department of Cardiology, District Hospital, Chojnice, Poland \\ ${ }^{2}$ Department of Cardiology and Electrotherapy, Medical University of Gdansk, Poland
}

A 73-year-old male was admitted for coronary angiography. A right distal radial approach, called the snuffbox approach, was chosen for the default access. A 6-French sheath was inserted after successful puncture by a 21 -gauge open needle with the anterior wall puncture technique. Angiography revealed moderate proximal left anterior descending artery stenosis, and after functional assessment a pharmacological treatment was recommended. Bandage compression was applied on the puncture site for $3 \mathrm{~h}$.

The next day hematoma and edema of the dorsal part of the hand was noticed (Fig. 1A). A gentle thrill was present on the puncture site in the anatomical snuffbox. Vascular ultrasonography demonstrated arteriovenous fistula (AVF) with one direction flow from the artery to vein (Fig. 1B, C; Suppl. Video 1). The double artery compression technique was performed for $10 \mathrm{~min}$, i.e. with si- multaneous ultrasound transducer on the anatomical snuffbox and manually on the traditional radial artery puncture site. Ultrasonography, at the time of compression, revealed only residual blood flow in the artery lumen. One month later, follow-up ultrasonography confirmed the absence of AVF and patent radial artery (Fig. 1D, Suppl. Video 1).

Since the first report of the snuffbox approach for coronary procedures in 2017 , it has become more widely used. Local vascular complications following traditional transradial access are wellrecognized: hematoma and occlusion occur regularly, other like pseudoaneurysm, perforation or AVF are quite exceptional. Complications of the distal radial approach are not yet fully defined.

Herein is illustrated the first case of puncture-induced AVF following the snuffbox approach, and efficacious management with radial flow preservation.

Conflict of interest: None declared

Address for correspondence: Lukasz Kozinski, MD, Department of Cardiology, District Hospital, ul. Leśna 10, 89-600 Chojnice, Poland, tel/fax: +48 5239566 39, e-mail: lukekozinski@gmail.com

Received: 18.07.2019 Accepted: 20.01.2020 


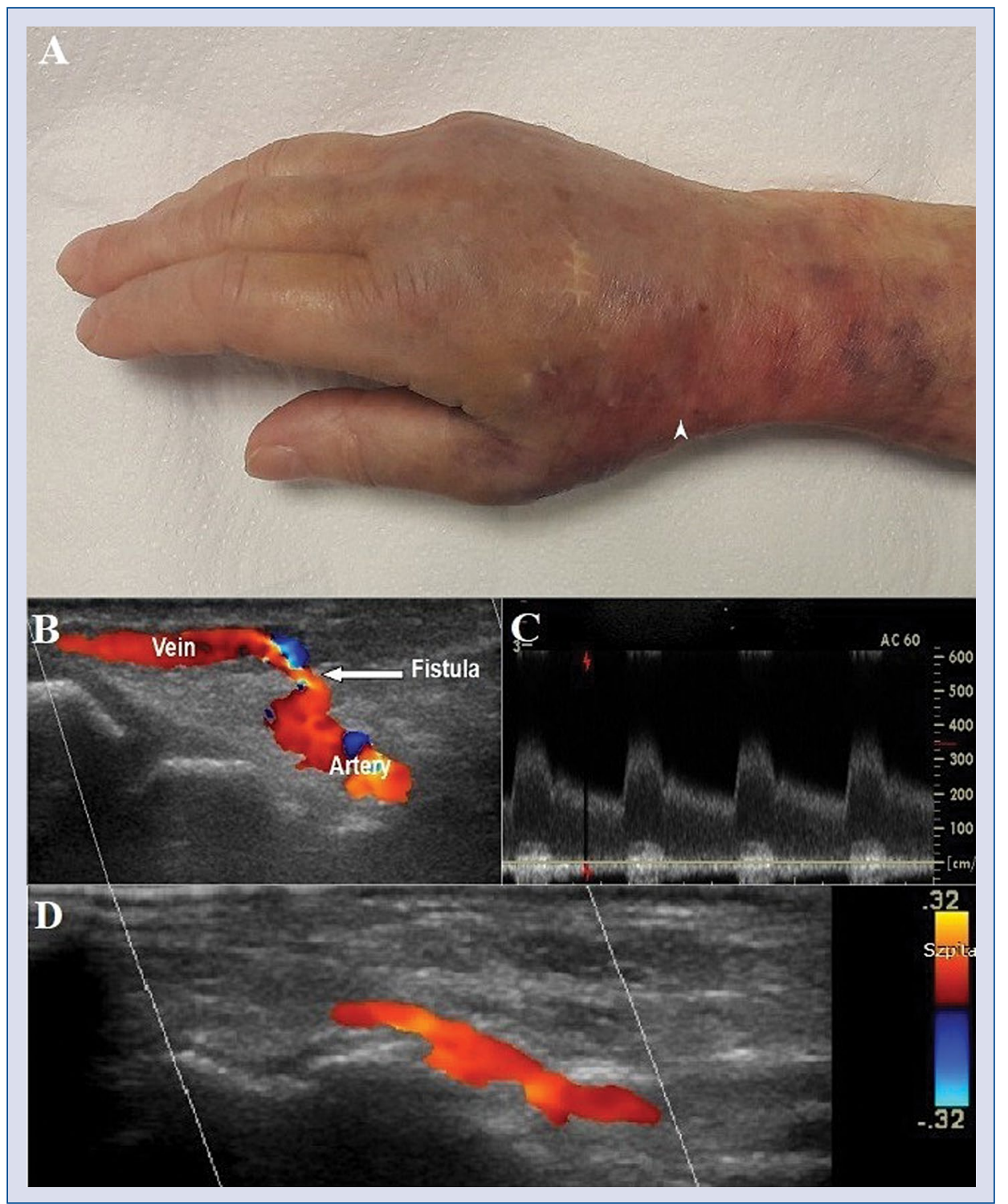

Figure 1. A. Hematoma and edema of the dorsal part of the hand presented the following day, post procedure. Ultrasound showing: B. Fistula between radial artery in the anatomical snuffbox and local superficial vein; C. One direction flow from artery to vein; D. Follow up ultrasonography: patent artery and the absence of arteriovenous fistula. Arrowhead in the panel $A$ indicates the puncture site of the distal radial artery. 